\title{
FIBROMA NASOFARINGEO JUVENIL: RELATO DE UM CASO COM INVASÃO DAS FOSSAS CRANIANAS ANTERIOR E MEDIA
}

\author{
Walter C. Pereira* \\ ALMIR F. ANDRADE ** \\ L. C. Mattosinho-França *** \\ ROLANDO A. TENUTO ****
}

O fibroma nasofaríngeo juvenil é um tumor relativamente raro na patologia otorrinolaringológica. Sua ocorrência oscila entre um e dois casos, em média, por ano, nos maiores serviços especializados do mundo $3,4,8,11,15$. Harma ${ }^{12}$, na Finlândia, calcula a existência de um fibroma nasofaríngeo juvenil para cada 6.000 pacientes com afecções otorrinolaringológicas. Contràriamente a êstes dados, verificados pela maioria dos autores, Acuña ${ }^{1}$, no México, observou 70 casos de fibroma nasofaríngeo durante apenas 6 anos.

O fibroma nasofaríngeo juvenil constitui entidade patológica extremamente curiosa, sendo duvidosa sua classificação entre as verdadeiras neoplasias em virtude de certas peculiaridades, que serão discutidas mais adiante. Trata-se de tumor de natureza benigna e não infiltrativo, que inicia seu crescimento nos planos profundos (fascia basal) da nasofaringe ou das coanas ${ }^{5}$ e se propaga às estruturas vizinhas, comprimindo-as sem, contudo, infiltrá-las. Embora não possua cápsula envolvente que o delimite dos tecidos adjancentes, o fibroma nasofaríngeo não os invade, destruindo-os por erosão ou deformando-os por efeito da expansão contínua. Assim, as fossas nasais, os seios maxilar, etmoidal e esfenoidal, ou mesmo a orofaringe, são progressivamente preenchidos e destruídos pela massa tumoral. No entanto, é excepcional que o fibroma nasofaríngeo ultrapasse os limites da base do crânio ${ }^{5}$.

Recentemente tivemos o ensejo de observar um fibroma nasofaríngeo juvenil com crescimento para o interior das fossas cranianas anterior e média. Durante todo o tempo de atividade dos Departamentos de Otorrinolaringologia e de Neurologia desta Faculdade foi o único caso em que tal fato ocorreu, embora o número de fibromas nasofaríngeos aqui observados atinja a casa dos quarenta ${ }^{16}$.

Departamento de Neurologia da Faculdade de Medicina da Universidade de São Paulo (Prof. Adherbal Tolosa): * Neurocirurgião; ** Estagiário; *** Patologista; **** Professor de Disciplina de Neurocirurgia. 
J.E.A., 15 anos de idade, sexo masculino, côr branca, registro H.C. 810103. O paciente foi admitido em 25-8-1966 com história iniciada há um ano e meio, caracterizada por dores ao nível da regiăo orbitária direita, perda sanguínea freqüente pela narina do mesmo lado e protusão do globo ocular direito. Estes sintomas e sinais foram se agravando lentamente, de modo particular as epistaxes, que se tornaram copiosas e quase contínuas. Nos antecedentes pessoais e familiares do paciente nada foi encontrado que pudesse estar relacionado com a moléstia atual. Wxame clínico-neurológico — Pressão arterial $120 \times 80$; pulso 80/min; temperatura $36,5^{\circ} \mathrm{C}$. Bom estado geral; mucosas coradas; gânglios não palpados; pêlos axilares e pubianos com distribuição peculiar ao sexo e caracteres sexuais secundarios normalmente desenvolvidos para a idade. Segmento cefálico: protusão acentuada do globo ocular direito, não sendo observados edema, engurgitamento venoso, sopros ou frêmitos em tôda a região orbitária. Fundos oculares normais. Exame rinoscópico: tumoração avermelhada de superfície irregular visivel na metade posterior da fossa nasal direita, empurrando o septo para o lado oposto; 0 tumor apresenta consistência fibrosa e sangra abundantemente ao mais leve toque. o exame dos demais aparelhos nada mostrou de anormal, inclusive do ponto de vista neurológico. Exames complementares - Craniogramas: aumento da densidade ao nivel dos andares médio e anterior, notando-se, no perfil, formação densa fazendo saliência acima do plano esfenoidal; a cavidade orbitária direita apresenta diâmetros aumentados e aspecto irregular do assoalho; abaulamento da fossa nasal direita com desvio acentuado do septo nasal para o lado oposto (fig. 1 A e B). Pneumencefalografia fraccionada: redução das dimensōes das cisternas infundibular e optoquiasmática provocada por imagem tumoral intracraniana de projeção órbitoesfenoidal (fig. 1 C). Carótido-angiografia direita: elevação discreta do segmento inicial da artéria cerebral média e desvio para trás do sifão carotídeo; a artéria maxilar interna é bem vizível e se dirige para a tumoração (fig. 1 D). Eletrencefalograma: moderada assimetria da atividade elétrica entre os hemisférios cerebrais, com menor amplitude e presença de ondas teta em proporção relativamente elevada no hemisfério cerebral direito. 17-cetosteróides na urina de 24 horas: 10,9 $\mathrm{mg}$. 17-hiaroxiesteróides na urina de 24 horas: $8,2 \mathrm{mg}$.

Em virtude da ausência de sinais de hiperestrogenismo, tanto clínicos como laboratoriais, foi feita biopsia do tumor nasal para a confirmação de um possível fibroma nasofaríngeo juvenil; a primeira tentativa, porém, revelou processo inflamatório crônico inespecífico. Uma segunda biopsia, feita alguns dias depois, retirando tecido mais profundamente situado, mostrou tratar-se realmente de um angiofibroma.

Como o tumor, em sua porção intracraniana, apresentasse relações intimas com a artéria carótida interna, decidiu-se iniciar sua excisão mediante craniotomia osteoplástica frontotemporal direita. Esta operação foi realizada em 28-11-1966, observando-se grande abaulamento da fossa craniana anterior, predominantemente no lado direito, que se continuava em direção à fossa craniana média direita. Não havia solução de continuidade da tábua óssea em local algum, porém esta se encontrava tão fina que a coloração vermelho-escura do tumor era bem visível através dela. Com o auxílio de pinça anatômica a tábua interna foi fàcilmente aberta e, ampliando-se êste orifício, procedeu-se à curetagem de tôda a massa tumoral accessível. Tratava-se de material mole e friável, havendo, contudo, pequenas áreas de consistência muito dura; o sangramento era intenso e de difícil contensão. O tumor preenchia os seios maxilar, etmoidal e, parcialmente, o esfenoidal do lado direito, propagando-se para o lado oposto através dos seios esfenoidal e etmoidal. Tôda a porção que se projetava para o interior do crânio foi retirada, sendo feita ulteriormente cuidadosa hemostasia com termocautério e "gel-foam". O pós-operatório decorreu sem complicações.

Uma semana depois o paciente foi novamente operado no Departamento de Otorrinolaringologia, sendo feita exérese do restante da neoplasia por via transmaxilar. O aspecto do tumor era o mesmo verificado durante a primeira inter- 

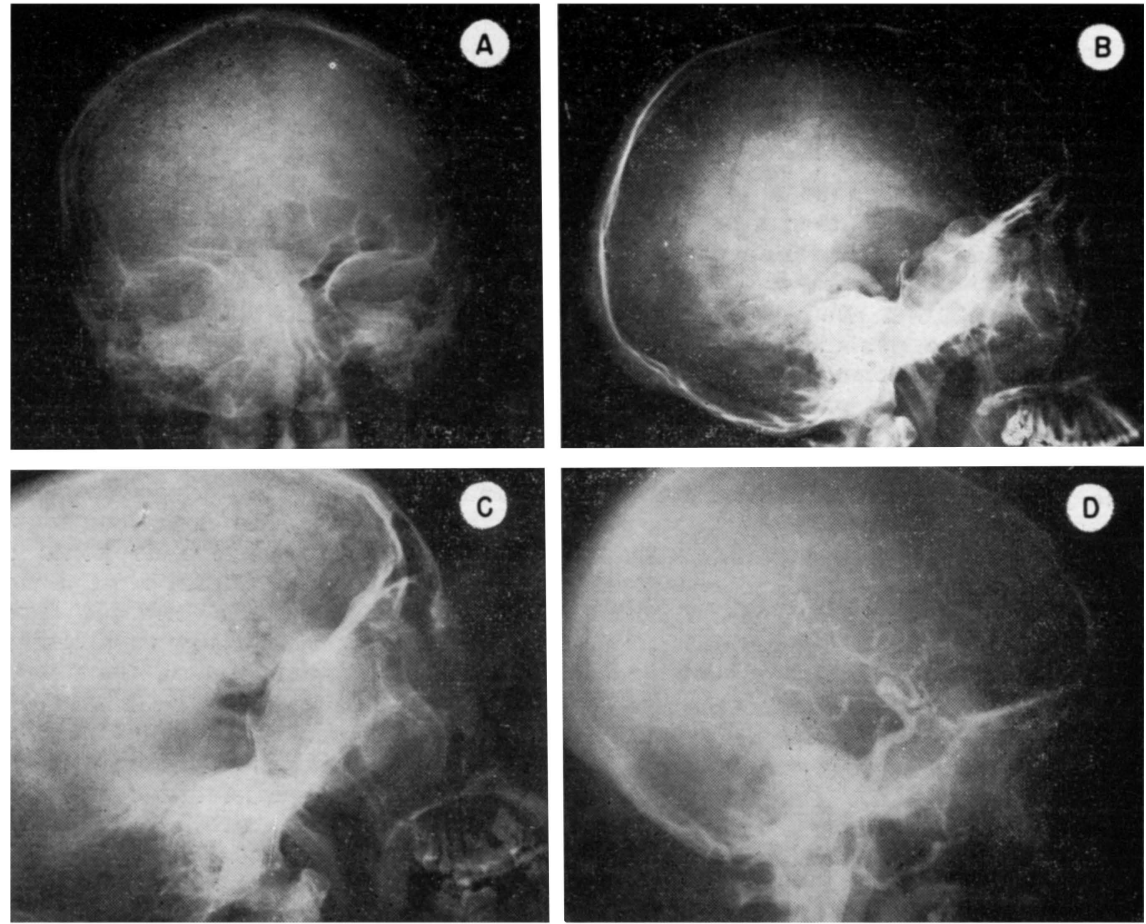

Fig. 1 - Caso J.E.A. Em A e B craniogramas mostrando volumoso tumor com densidade de partes moles, fazendo saliência acima do plano esfenoidal; a cavidade orbitária direita está com os diâmetros alargados e apresenta aspecto irregular do assoalho; abaulamento da fossa nasal direita com desvio do septo para a esquerda. Em $C$, pneumencefalografia fracionada mostrando redução das cisternas infudibular $e$ optoquiasmática. Em $D$, carótido-angiografia direita mostrando desvio do sifão carotídeo para trás e participação da artéria maxilar interna na circulação do tumor.

venção, confirmando-se seu caráter não infiltrativo, pois em tôda a periferia o pano de clivagem era muito nitido. A neoplasia ocupava inteiramente os seios maxilar e etmoidal direitos, invadindo a fossa nasal e empurrando o septo para a esquerda e a parede infra-orbitária para cima; o cavum estava também quase totalmente ocupado pelo tumor. A zona de origem do processo não foi identificada. A massa tumoral foi totalmente retirada, sendo a hemostasia feita com facilidade, usando-se termocautério e "gel-foam". o pós-operatório também desta vez decorreu sem qualquer complicação.

O exame histopatológico do material obtido nas duas operações suscitou controvérsia, pois os cortes, examinados por diferentes especialistas, levaram aos diagnósticos de angiofibroma e de sarcoma osteogênico. No entanto, o exame de maior número de fragmentos do tumor, associado ao quadro clínico e à observação cirúrgica de que o processo não era infiltrativo, permitiram o diagnóstico definitivo de fibroma nasofaringeo juvenil (fig. 2).

Após o tratamento cirúrgico houve desaparecimento total da sintomatologia. Um ano depois o paciente continuava assintomático, não tendo os exames rinoscópico e radiológico mostrado qualquer indicio de recidiva do tumor. 

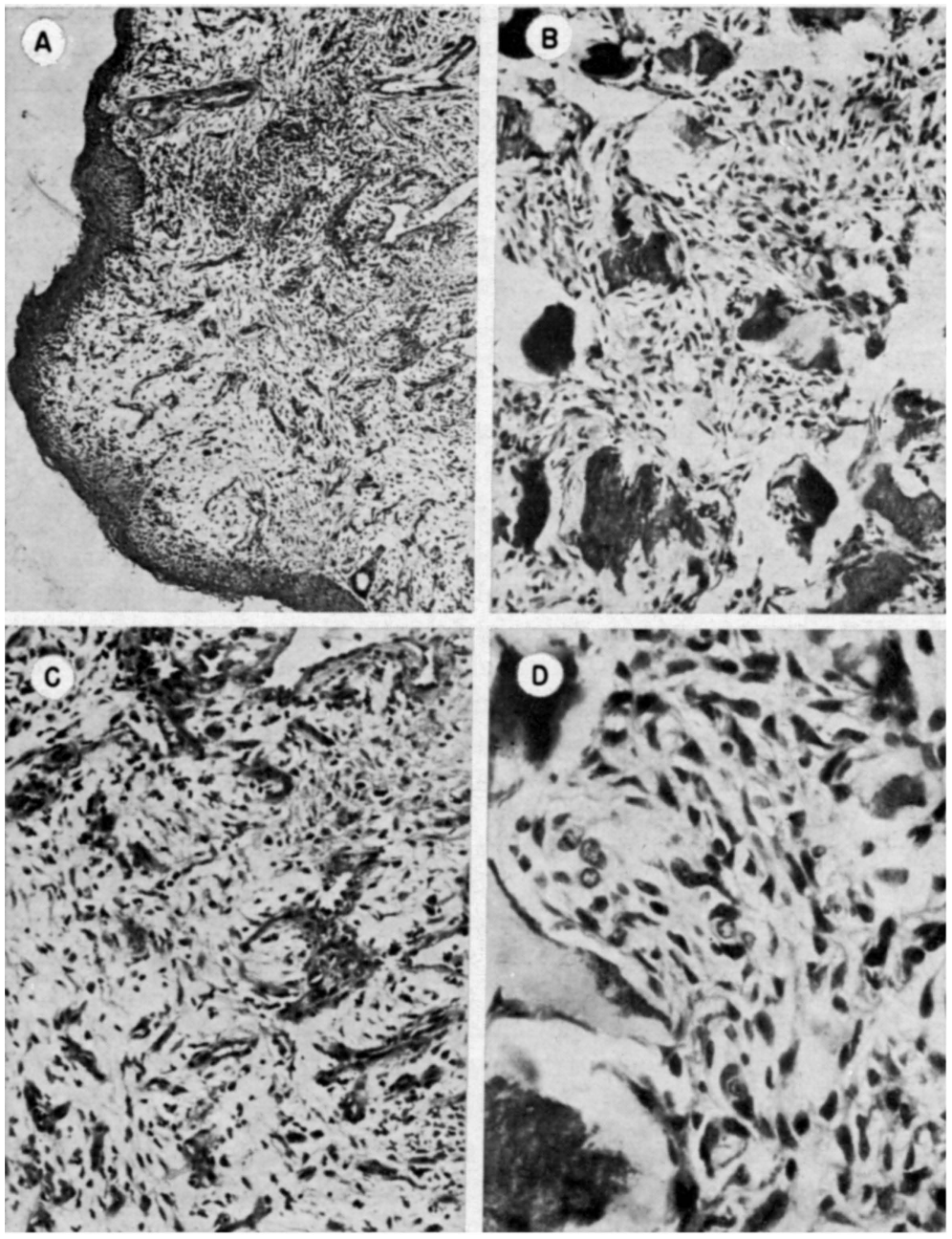

Fig. 2 - Caso J.E.A. Microfotografias da peça cirúrgica (coloração $H . E.):$ em $A$, revestimento epitelial e estroma do tumor; em $B$, com maior aumento, vê-se a profundidade do estroma, mostrando metaplasia óssea; em $C$, observa-se ainda a profundidade do estroma com tecido conjuntivo frouxo e grande número de vasos capilares; em $D$, com grande aumento, vê-se um aspecto particular da metaplasia óssea no estroma.

COMENTARIOS

Nenhum dos tumores benignos da nasofaringe é tão típico e conhecido em suas peculiaridades como o fibroma juvenil ${ }^{3}$. Ocorre quase exclusivamente em adolescentes do sexo masculino (entre os 10 e 20 anos), apresentando tendência à regressão espontânea quando o indivíduo ultrapassa a 
puberdade. Os fibromas da nasofaringe descritos em crianças menores ou em pacientes idosos, assim como em mulheres ${ }^{10,17}$, diferem muito no comportamento e não devem, portanto, ser agrupados com o fibroma nasofaríngeo genuino ${ }^{3,17}$. Tais tumores deveriam ser classificados, na maioria dos casos, como fibromas inespecíficos, fibromas pediculados ou polipos vascularizados ${ }^{3}$.

A característica mais curiosa do fibroma nasofaríngeo juvenil é a tendência que apresenta de involuir espontâneamente após a puberdade. Esta peculiaridade, segundo Martens e Mac Pherson ${ }^{14}$, relaciona êste tumor aos granulomas da gravide z e piogênico e à carúncula uretral. Tal característica torna problemática a inclusão do fibroma nasofaríngeo juvenil entre as verdadeiras neoplasias benignas, devendo, isto sim, ser considerado uma hiperplasia reativa do tecido colágeno, a qual sofreria involução após a cessação de um possivel estímulo genético ${ }^{3,5}$.

Martin e col. ${ }^{15}$ realçaram a importância do fator hormonal na etiopatogenia do fibroma nasofaríngeo juvenil. Êstes autores observaram franca involução dêste tumor após o desenvolvimento pleno dos caracteres sexuais secundários, espontâneamente ou acelerado com medicamentos; baseados neste fato sugerem que a causa primária desta afecção seja o déficit de andrógenos ou o excesso de estrógenos. Lismonde e Lepage ${ }^{13}$ aventaram três hipóteses etiopatogênicas: a) reação perióstica exacerbada em conseqüência à irritação crônica provocada por infecção das adenóides; b) anomalias do desenvolvimento da hipófise; c) disfunção tímica. Osborn (cit. por Berendes e col. ${ }^{3}$ ) considera o fibroma nasofaríngeo como sendo uma malformação de tecido eréctil de caráter semelhante ao harmartoma. No caso que ora apresentamos não havia sinais clínicos de hiperestrogenismo e a dosagem dos 17-ceto e oxiesteróides na urina de 24 horas revelou valores normais. Portanto, em nosso paciente o fator hormonal parece não ter atuado, pelo menos de maneira preponderante, no desencadeamento e na manutenção do processo.

Do ponto de vista morfológico o fibroma nasofaríngeo juvenil assemelha-se aos fibromas em geral. É constituido principalmente de tecido colágeno; muitas vêzes, porém, há produção de escassa quantidade de fibras colágenas, predominando os fibroblastos de conformação variada e células gigantes polinucleadas, consideradas como fibroblastos com vários núcleos ${ }^{3}$. Nos fibromas nasofaríngeos de evolução mais longa são observados sinais de regressão, que se seguem a oclusões vasculares: degenerações mixomatosa, hialina e esclerosa distribuídas em áreas mais ou menos extensas. Êstes sinais degenerativos se acentuam após a hormonoterapia com andrógenos ${ }^{18}$. O componente vascular destas neoplasias é também importante: encontramse espaços vasculares numerosos com dimensões variadas, sendo muito irregulares na periferia do tumor. Observam-se freqüentemente lacunas venosas com paredes delgadas, constituídas, em geral, apenas pelo endotélio; estas lacunas são comprimidas pelos tecidos adjacentes, tomando o aspecto de fendas. Em muitos campos do tumor pode ser observada proliferação vascular; nesses locais as células adquirem, por vêzes, o aspecto de elemen- 
tos mesenquimatosos embrionários, podendo levar à confusão com processos malignos se o exame se limitar a tais áreas. Estas células indiferenciadas, que constituem pequenos espessamentos das paredes vasculares, foram consideradas como angioblastos por Harma ${ }^{12}$. Em virtude de sua riqueza vascular êste tumor é também chamado angiofibroma. Na periferia da neoplasia não se encontra uma verdadeira cápsula; é comum, no entanto, a presença de processo inflamatório crônico provocado pelas hemorragias freqüentes. O tumor, durante seu crescimento, vai envolvendo os tecidos vizinhos, sendo freqüentemente encontradas pequenas esquírulas ósseas em seu interior. Os aspectos histológicos descritos permitem interpretar as discrepâncias nos diagnósticos histológicos do caso que relatamos. A primeira biopsia foi feita muito superficialmente e apenas demonstrou o processo inflamatório crônico da periferia do tumor; casos semelhantes são referidos por Correa e Lima ${ }^{5}$. Quanto ao diagnóstico de sarcoma osteogênico, feito após o exame do material retirado durante a exérese transmaxilar do tumor, possìvelmente êste foi sugerido por algumas áreas da neoplasia que, como já assinalamos, podem apresentar aspecto indiferenciado. No entanto, a maior parte do tumor se apresentava com características histológicas de benignidade, não havendo metástases nem infiltração dos tecidos adjacentes.

Quanto à sintomatologia, o fibroma nasofaríngeo juvenil determina com freqüência o mesmo quadro apresentado por nosso paciente, acrescido, em alguns casos, de obstrução nasal e complicações infecciosas por bloqueio dos seios paranasais. Quando êste fato ocorre pode surgir adenopatia satélite, induzindo, às vêzes, à suspeita de neoplasia maligna. Embora no caso aqui relatado não estivessem presentes os sinais de hiperestrogenismo, êstes são freqüentemente registrados.

O diagnóstico é feito pelo estudo radiológico do crânio e seios paranasais, que permite localizar a massa tumoral, a qual se apresenta sempre como imagem com densidade de partes moles. A angiografia pela carótida comum, ou externa, permite observar a vascularização do tumor pela artéria maxilar interna, que geralmente se apresenta calibrosa ${ }^{3}$. No caso por nós estudado o exame radiológico do crânio, inclusive a pneumencefalografia fraccionada, demonstrou claramente a invasão das fossas cranianas anterior e média pela neoplasia. A dosagem dos 17-cetosteróides urinários costuma mostrar valores muitos baixos ${ }^{15}$; em nosso paciente tal fato não foi observado. Carbonara e Salonna (cit. por Berender e col. ${ }^{3}$ ) estudaram a eliminação dos 17-cetosteróides, 11-oxiesteróides, esteróides fenólicos e da gonadotropina em doentes com fibroma nasofaríngeo; sòmente encontraram alterações nitidas da gonadotropina, que se encontrava aumentada, deduzindo que nesta afecção ocorre atividade hipofisária elevada com insuficiência gonadal. A biopsia deve ser indicada com reservas em virtude da natureza altamente sangrante do tumor ${ }^{9}$; no entanto ela poderá ser feita quando o diagnóstico fôr duvidoso.

O diagnóstico diferencial deve ser estabelecido com o fibrossarcoma (muitíssimo raro); fibroma simples ou outros tumores nodulares da região 
e polipos indurados e vascularizados secundàriamente ${ }^{3}$. Além disso devem ser lembrados os osteomas e meningeomas nos raríssimos casos em que o processo invade a base do crânio.

A maioria dos autores advoga conduta cirúrgica precoce para o tratamento do fibroma nasofaríngeo juvenil. A ligadura prévia da artéria carótida externa é, muitas vêzes, indicada para reduzir o sangramento durante a exérese. A radioterapia é também considerada de utilidade como tratamento exclusivo, a fim de reduzir o volume da neoplasia antes da exérese cirúrgica ou para a complementação desta quando a retirada do tumor não foi total. Alguns fibromas nasofaríngeos são, contudo, absolutamente insensiveis à radioterapia ${ }^{3}$. Quanto ao tratamento hormonal com andrógenos, a maioria dos autores afirma que êste tem apenas efeito inibidor sôbre as hemorragias e, talvez, também retarde o crescimento do tumor 2,3, 7, 18; outros, contudo, o consideram totalmente inútil ${ }^{1}$.

Em relação ao prognóstico, o fibroma nasofaríngeo juvenil comporta-se como neoplasia benigna, observando-se cura na grande maioria dos doentes tratados com cirurgia radical ${ }^{5,6}$.

\section{R E S M O}

O fibroma nasofaríngeo juvenil é um dos tumores mais raramente encontrados e sua propagação para o interior do crânio é excepcional. A etiopatogenia desta afecção é discutida, não sendo considerada por muitos autores uma verdadeira neoplasia benigna, mas sim uma hiperplasia reativa do tecido colágeno causada por estímulo genético ou hormonal.

Relatamos um caso de fibroma nasofaríngeo juvenil em adolescente masculino de 15 anos de idade, o qual se propagava para as fossas cranianas anterior e média. $O$ doente não apresentava sinais clínicos ou laboratoriais de hiperestrogenismo. Foi operado em dois tempos, sendo feita inicialmente exérese da porção intracraniana do tumor mediante craniotomia frontotemporal e, uma semana depois, o restante foi retirado por via transmaxilar. Um ano após o tratamento cirúrgico o paciente foi re-examinado, não havendo sinais clínicos ou radiológicos de recidiva do processo.

\section{S U M M A R Y}

Juvenile nasopharyngeal fibroma with involvement of the anterior
and middle cranial fossae: a case report.

The juvenile nasopharyngeal fibroma is one of the less frequently found tumors and its propagation towards the skull cavity is very rare. The etiopathogeny of this process is controversial. Many authors do not consider it a true beningn neoplasm, but a reactive hyperplasia of the collagen tissue caused by a genetical or hormonal stimulus. 
A case of juvenile nasopharyngeal fibroma in a 15-year-old male is reported. The tumor extended to the anterior and middle fossae. The patient failed to show any clinical or laboratory sign of hyperestrogenism. The operation was performed in two stages: firstly the removal of the intracranial portion of the growth was performed through a fronto-temporal craniotomy; one week later, the remaining was excised through a trasmaxillar approach. One year after the surgical treatment the patient was re-evaluated and no clinical or radiological signs of the tumor recurrence were evidenced.

R E F E R E N C I A S

1. ACUNA, R. T. - The nasopharyngeal fibroma and its treatment. Arch. Otolaryng. (Chicago) 64:451, 1956.

2. AURIN, A.; GANDON, J. \& BURGEAT, M. - Que peut-on attendre du traitment hormonal des fibromes nasopharyngiens. Ann. Oto-laryng. (Paris) 73: $301,1956$.

3. BERENDES, J.; LINK, R. \& ZOLLNER, F. - Tratado de Otorrinolaringologia. Trad. por J. D. Vazquez. Editorial Cientifico-Medica, Barcelona, 1969, pp. 620-628.

4. BRUNNER, H. - Nasopharyngeal fibroma. Ann. Otol. (St. Louis) 60:29, 1942.

5. CORREA, A. \& LIMA, M. L. M. T. - Fibroma nasofaríngeo juvenil. Rev. paul. Med. 43:415, 1953.

6. CORREA, A. \& PAIVA, L. - Tumores primitivos da rinofaringe em crianças. Rev. Hosp. Clin. 5:363, 1958.

7. DElarue, J.; PAIllas, H.; PAYEN, J. \& BURgeat, M. - Les fibromes nasopharyngiens: e`udes anatomo-pathologiques. Sem. Hôp. Paris 32:3801, 1956.

8. FIGI, F. A. - Fibromas of the nasopharynx. J. Amer. med. Ass. 115:665, 1940.

9. FIGI, F. A. \& DAVIS, R. E. - The management of nasopharyngeal fibroma. Laryngoscope (St. Louis) 60:794, 1950.

10. FINNERMAN, W. B. - Juvenile nasopharyngeal angiofibroma in the female. Arch. Otolaryng. 54:620, 1951.

11. HARKNEN, M. \& MALMIO, K. - On the treatment of nasopharyngeal fibroma and its results. Acta oto-laryng. (Stockholm) SuppI. 67:33, 1948.

12. HARMA, R. A. - Nasopharyngeal angiobroma. Acta oto-laryng. (Stockholm) Suppl. 146:1-74, 1958.

13. LiSMONDE, G. \& LEPAGE, G. - A propos de deux cas de fibrome nasopharyngiens: considerations thérapeutiques. Acta neurol. psychiat. belg. 58:848, 1958.

14. MARTENS, V. E. \& Mac PHERSON, D. I. - Fibroangioma: a proposed descriptive term for granuloma pyogenicum, granuloma gravidarum, juvenile angiofibroma and erethral caruncle. Arch. Path. (Chicago) 61:120, 1956. 
348 ARQ. NEURO-PSIQUIAT. (SAO PAULO) VOL. 27, N. 4, DEZEMBRO, 1969

15. MARTIN, H.; ERLICH, H. E. \& ABELS, J. C. - Juvenile nasopharyngeal angiofibroma. Ann. Surg. 127:513, 1948.

16. NOVA, R. da - Comunicação pessoal.

17. PARCHET, V. - L'angiofibrome nasopharyngien chez la femme. Ann. Otolaryng. (Paris) 68:60, 1951.

18. SCHIFF, M. - Juvenile nasopharyngeal angiofibroma: a theory of pathogenesis. Laryngoscope (St. Louis) 69:981, 1959.

Departamento de Neurologia - Faculdade de Medicina da Universidade de São Paulo - Caixa Postal 3461 - São Paulo, SP - Brasil. 